\title{
Type 2 innate lymphoid cells treat and prevent acute gastrointestinal graft-versus-host disease
}

\author{
Danny W. Bruce, ${ }^{1}$ Heather E. Stefanski, ${ }^{2}$ Benjamin C. Vincent, ${ }^{1,3,4}$ Trisha A. Dant, ${ }^{5}$ Shannon Reisdorf, ${ }^{1}$ Hemamalini Bommiasamy, \\ David A. Serody, ${ }^{1}$ Justin E. Wilson, ${ }^{1,4}$ Karen P. McKinnon, ${ }^{1,5}$ Warren D. Shlomchik, ${ }^{6}$ Paul M. Armistead, ${ }^{1,7}$ Jenny P.Y. Ting, ${ }^{1,4}$ \\ John T. Woosley, ${ }^{8}$ Bruce R. Blazar, ${ }^{2}$ Dietmar M.W. Zaiss, ${ }^{9}$ Andrew N.J. McKenzie, ${ }^{10}$ James M. Coghill, ${ }^{1,3}$ and Jonathan S. Serody ${ }^{1,3,5}$ \\ ${ }^{1}$ Lineberger Comprehensive Cancer Center, University of North Carolina at Chapel Hill, Chapel Hill, North Carolina, USA. ²Department of Pediatrics and University of Minnesota Cancer Center, Minneapolis, \\ Minnesota, USA. ${ }^{3}$ Department of Medicine, ${ }^{4}$ Department of Genetics, and ${ }^{5}$ Department of Microbiology and Immunology, University of North Carolina at Chapel Hill, Chapel Hill, North Carolina, USA. \\ ${ }^{6}$ Department of Medicine and Cancer Center of the University of Pittsburgh, Pittsburgh, Pennsylvania, USA. 'Department of Chemistry and ${ }^{8}$ Department of Laboratory Medicine and Pathology, University \\ of North Carolina at Chapel Hill, Chapel Hill, North Carolina, USA. 'entre for Immunity, Infection and Evolution, and the Institute for Immunology and Infection Research, School of Biological Sciences, \\ University of Edinburgh, Edinburgh, United Kingdom. ${ }^{10}$ The MRC Laboratory of Molecular Biology, Cambridge, United Kingdom.
}

\begin{abstract}
Acute graft-versus-host disease (aCVHD) is the most common complication for patients undergoing allogeneic stem cell transplantation. Despite extremely aggressive therapy targeting donor T cells, patients with grade III or greater aCVHD of the lower GI tract, who do not respond to therapy with corticosteroids, have a dismal prognosis. Thus, efforts to improve understanding of the function of local immune and non-immune cells in regulating the inflammatory process in the $\mathrm{Cl}$ tract during aGVHD are needed. Here, we demonstrate, using murine models of allogeneic BMT, that type 2 innate lymphoid cells (ILC2s) in the lower GI tract are sensitive to conditioning therapy and show very limited ability to repopulate from donor bone marrow. Infusion of donor ILC2s was effective in reducing the lethality of aCVHD and in treating lower GI tract disease. ILC2 infusion was associated with reduced donor proinflammatory Th1 and Th17 cells, accumulation of donor myeloid-derived suppressor cells (MDSCs) mediated by ILC2 production of IL-13, improved GI tract barrier function, and a preserved graftversus-leukemia (CVL) response. Collectively, these findings suggest that infusion of donor ILC2s to restore gastrointestinal tract homeostasis may improve treatment of severe lower GI tract aGVHD.
\end{abstract}

\section{Introduction}

Allogeneic stem cell transplant (allo-SCT) has the potential to provide curative therapy for patients with high-risk acute leukemia, lymphoid malignancies, and other malignant diseases (1-3). Despite improvements in HLA typing and stem cell donor selection, graft-versus-host disease (GVHD) remains the major complication of allo-SCT, with incidence of acute GVHD (aGVHD) ranging from $30 \%$ to $80 \%$ and accounting for $15 \%-30 \%$ of mortality of transplant recipients $(4,5)$. Grade III-IV aGVHD involving the lower gastrointestinal tract is the most common cause of morbidity and mortality from aGVHD. The administration of corticosteroids is the standard approach for the treatment of patients with grade II-IV aGVHD, with approximately $70 \%$ of patients treated responding (6). However, the long-term survival of patients with corticosteroid-nonresponsive aGVHD involving the lower GI tract is dismal, with less than $20 \%$ of those patients alive 1 year after diagnosis (7). Clearly, new forms of therapy are needed for the

\footnotetext{
Note regarding evaluation of this manuscript: Manuscripts authored by scientists associated with Duke University, The University of North Carolina at Chapel Hill, Duke-NUS, and the Sanford-Burnham Medical Research Institute are handled not by members of the editorial board but rather by the science editors, who consult with selected external editors and reviewers.

Conflict of interest: The authors have declared that no conflict of interest exists.

Submitted: November 17, 2016; Accepted: February 2, 2017

Reference information: J Clin Invest. 2017;127(5):1813-1825

https://doi.org/10.1172/JCI91816.
}

treatment of patients with corticosteroid-nonresponsive aGVHD of the lower GI tract.

Research over the past 40 years has primarily focused on the role of donor-derived T cells in the pathogenesis of aGVHD (8). Work from both preclinical transplant models and clinical transplant studies has indicated a critical role for $\mathrm{T}$ cells, specifically Th1/Tc1 T cells, in the pathophysiology of aGVHD. Thus, treatment of aGVHD has almost entirely focused on targeting donor $\mathrm{T}$ cells. However, despite highly potent therapy targeting T cells such as alemtuzumab, outcome for patients with corticosteroid-nonresponsive aGVHD has not improved (7). This has led to increasing interest in the role of other proinflammatory immune cells, such as macrophages, neutrophils, and B lymphocytes, in the pathophysiology of aGVHD, and the local function of antiinflammatory immune and non-immune cells $(9,10)$.

A second group of immune cells that diminish the effector function of proinflammatory immune cells may be critical to the immune response during aGVHD. FoxP3-expressing Tregs limit the expansion and effector function of donor T cells. Infusion of donor Tregs has been shown to be an effective prophylactic approach for the prevention of aGVHD (11). At this time, it is not clear whether the infusion of Tregs can effectively treat ongoing aGVHD. Our group has shown that IL-13-activated bone marrow myeloid-derived suppressor cells (MDSCs) used at the time of bone marrow transplant (BMT) in preclinical models inhibited GVHD lethality (12). However, their ability to treat active aGVHD 

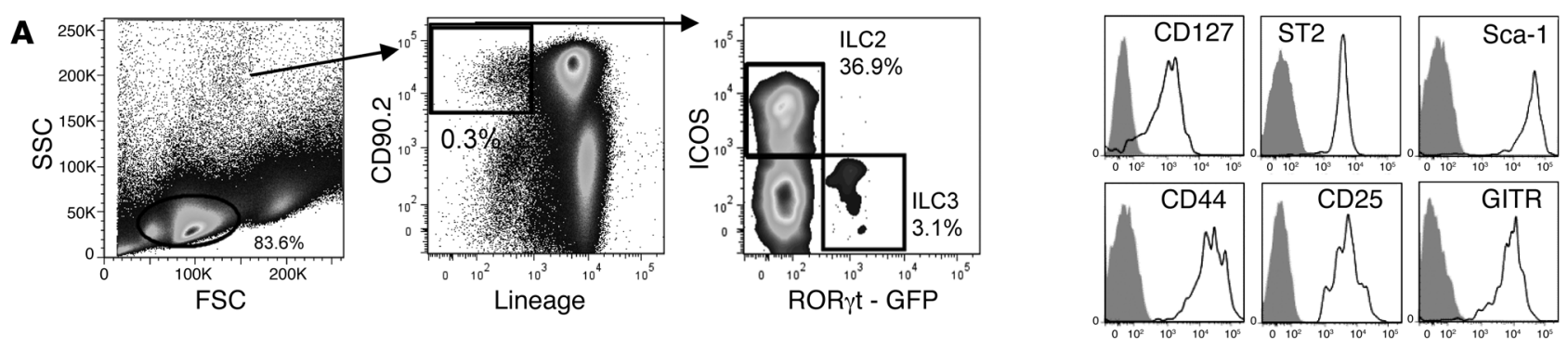

B
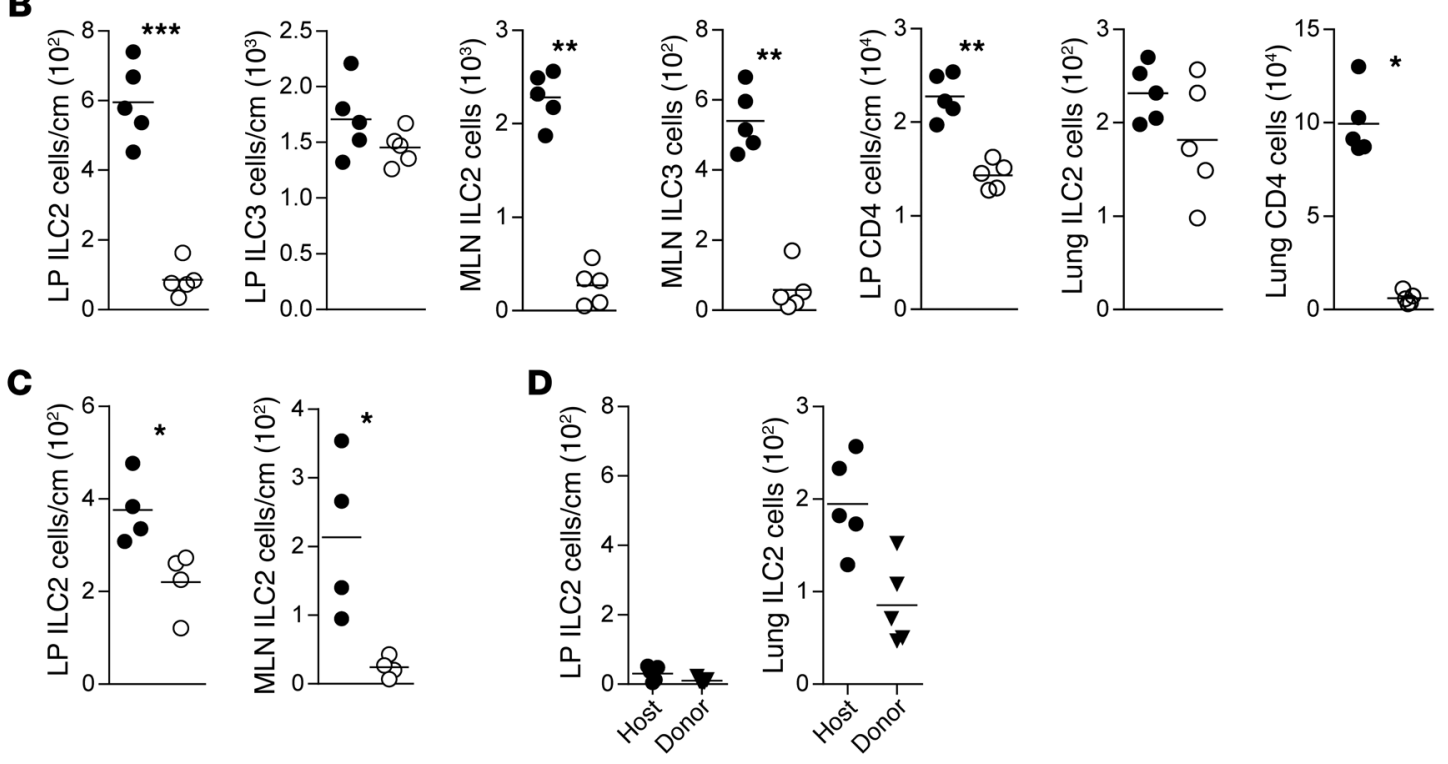

Figure 1. ILC2s are sensitive to radiation and chemotherapy conditioning. (A) Flow cytometry gating scheme used to define ILC2 and ILC3 subsets in MLN. (B) Quantitation by flow cytometry of innate lymphoid and CD4 ${ }^{+} T$ cells in the LP, MLN, or lung in mice without irradiation (filled circles) or 24 hours after receiving radiation (950 cGy) (open circles). Results represent 3 independent experiments; $n=5$ for each group. (C) Quantitation of ILC2s in the LP and MLN in mice receiving no therapy (filled circles) or 24 hours after receiving $200 \mathrm{mg} / \mathrm{kg}$ of cyclophosphamide (open circles). Results represent 2 independent experiments; $n=4$ for each. (D) Quantitation of host and donor-derived ILC2s in the LP and lung 28 days after BMT; mean \pm SEM. Results represent 2 independent experiments; $n=5$. Statistical analysis by Student's $t$ test with Welch's correction, ${ }^{* *} P<0.001,{ }^{* *} P<0.01,{ }^{*} P<0.05$.

is quite modest. Thus, despite intense research evaluating the function of immune cells that diminish effector $\mathrm{T}$ cell function, there is not a currently identified population of cells that has significant activity treating active aGVHD.

Over the past decade, a number of researchers have identified populations of innate immune cells (ILCs) critical for rapid mucosal immune responses $(13,14)$. The initial ILC described 4 decades ago was the NK cell. Recently, multiple populations of ILCs that generate IFN- $\gamma$ (ILC1), IL-5 and IL-13 (ILC2), and IL-17 and/or IL-22 (different subpopulations of ILC3s) have been described (15). Like Th2 cells, ILC2s - previously termed nuocytes or innate helper type 2 cells - express GATA-3 and ID-2. They generate substantial type 2 cytokines. ILC2s, which respond to IL-25, are critical to the anti-helminth immune response and play an important role in allergen-induced inflammation (16-20). The role of innate cells in the biology of aGVHD has been evaluated recently. Hanash et al. demonstrated that ILC3s in the GI tract were not sensitive to conditioning therapy but were decreased in mice with acute GVHD. The loss of ILC3s was associated with decreased generation of IL-22, impaired epithelial barrier function, and diminished numbers of intestinal stem cells (21). In humans, patients with decreased numbers of circulating CD69 ${ }^{+}$ILC2s and ILC3s had an increased risk of aGVHD (22).

We hypothesized that unlike ILC3s, there was a population of innate lymphoid cells that was sensitive to conditioning therapy. Here, we demonstrate that ILC2s in the GI tract but not in the lung are highly sensitive to conditioning therapy prior to allo-SCT and, more importantly, that there is a quite limited repopulation of ILC2s in the GI tract from donor bone marrow. Infusion of IL-33activated donor ILC2s significantly reduced GI tract-associated aGVHD mortality, improving GVHD scores and associated weight loss in two separate murine models. Additionally, ILC2 treatment of recipients with established aGVHD significantly increased survival. Finally, we provide a previously unreported mechanism by which ILC2s recruit donor myeloid-derived suppressor cells (MDSCs) to the lower GI tract to mitigate aGVHD. These studies strongly suggest that expanded ILC2s may be a potent cellular therapy for the treatment of lower GI tract aGVHD.

\section{Results}

Type 2 innate lymphoid cells are radiation sensitive. To evaluate the effect of conditioning therapy on ILCs, we irradiated B6D2 mice 
A

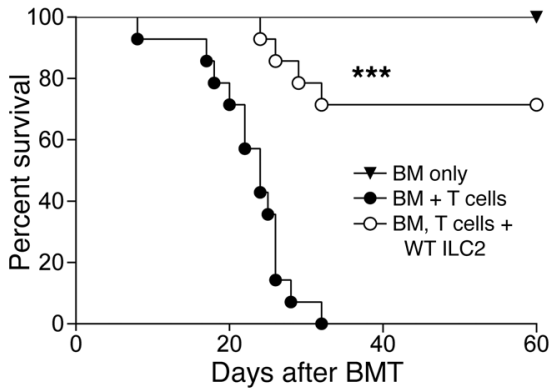

E

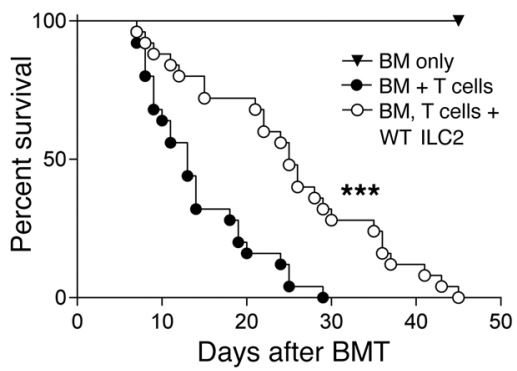

B

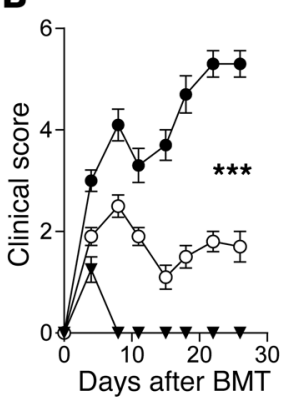

C

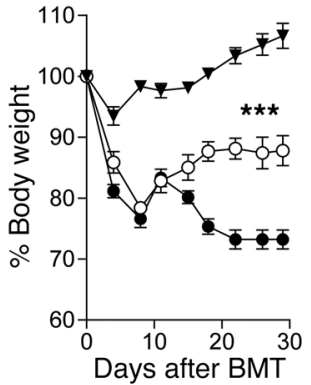

D

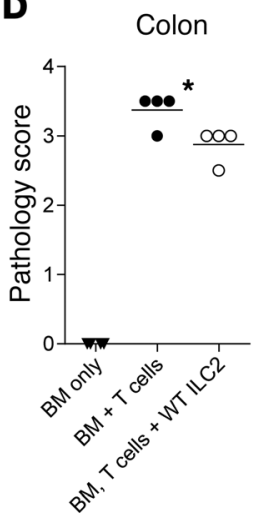

Spleen

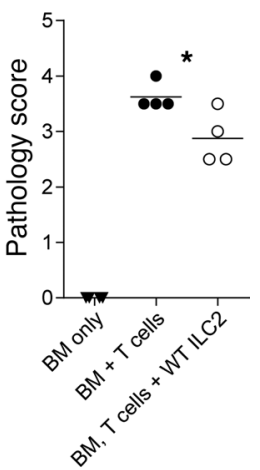

$\mathbf{F}$

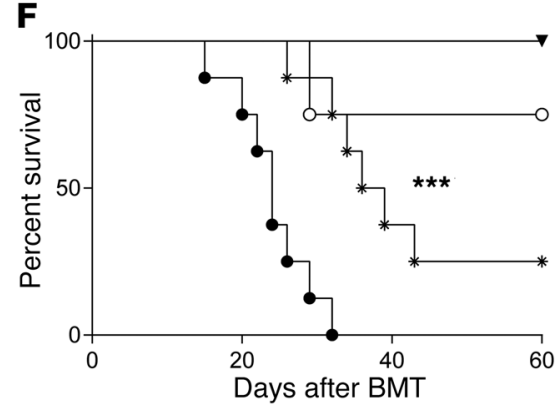

G
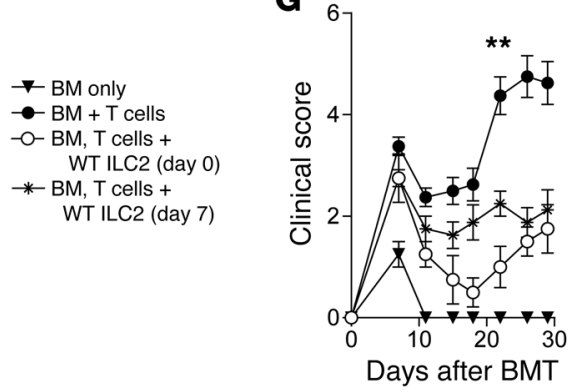

Figure 2. Co-transplantation of donor ILC2s reduces aCVHD incidence. Lethally irradiated B6D2 mice received TCD BM (BM only), BM plus total splenic T cells (BM + T cells), or BM plus T cells with activated ILC2s (BM, T cells + WT ILC2). (A) Kaplan-Meier plot of survival following allo-SCT; 1 representative of 3 experiments shown ( $n=8$ each experiment). Log-rank (Mantel-Cox) test, ${ }^{* *} P<0.001$. (B) Clinical score and (C) percentage of body weight change after transplantation. Two-way ANOVA, with Bonferroni correction for repeated measures of multiple comparisons, ${ }^{* * *} P<0.001$. (D) Pathology scores of histological evaluations of GVHD target organs, 20 days after BMT ( $n=4$ mice per group). Student's $t$ test with Welch's correction, ${ }^{*} P<0.05$. (E) Kaplan-Meier plot for irradiated BALB/c mice that received TCD BM (BM only), BM plus splenic T cells (BM + T cells), or BM plus T cells with ILC2s (BM, T cells + WT ILC2). Results represent 2 independent experiments; $n=14$. Log-rank (Mantel-Cox) test, ${ }^{* *} P<0.001$. (F) Kaplan-Meier plot for B6D2 recipients that received BM alone (BM only), BM and splenic T cells (BM + T cells), and WT ILC2s either at the time of transplant (BM, T cells + WT ILC2 [day 0]) or 7 days after transplant (BM, T cells + WT ILC2 [day 7]); data represent 2 experiments; $n=12$ total in each group. Log-rank (Mantel-Cox) test, ${ }^{* * *} P<0.001$. (G) Clinical score of recipients from Figure 2F. Two-way ANOVA, with Bonferroni correction for repeated measures of multiple comparisons, ${ }^{* *} P<0.01$.

(see Methods) and quantitated GI tract ILCs. ILC3s in the lamina propria (LP) of the colon but not the mesenteric lymph node (MLN), isolated 24 hours after lethal irradiation, were relatively resistant to radiation (Figure 1, A and B) $(13,21,23)$. In contrast, $\mathrm{CD} 4^{+} \mathrm{T}$ cells in the LP and lung were radiation sensitive. Unlike ILC3s, ILC2s from the LP of the colon were highly sensitive to radiation (Figure 1B). Similar to ILC3s, ILC2s in the MLN and spleen were exquisitely sensitive to radiation (Figure 1B and Supplemental Figure 1A; supplemental material available online with this article; https://doi.org/10.1172/JCI91816DS1). Interestingly, lung ILC2s were relatively resistant to radiation therapy (Figure 1B). We also examined the effects of chemotherapy on ILC2s and found that they were significantly reduced within 24 hours of cyclophosphamide treatment in the LP and MLN (Figure 1C).

Next, we evaluated the reconstitution of murine donor and recipient ILC2s 4 weeks after allo-SCT. Over this time period, there were extremely limited numbers of donor and/or recipient ILC2s in the LP of recipient mice that received irradiation and bone marrow without donor T cells, with a decrease in ILC2s in the LP at day 28 compared with day 1 (Figure 1D). By comparison, there were approximately 20-fold greater numbers of donor and/ or host ILC2s in the lung on day 28 after transplant (Figure 1D).
Thus, ILC2s in the GI tract, but not the lung, are sensitive to conditioning therapy and have extremely limited repopulation kinetics from donor bone marrow in the first 4 weeks after transplant.

Co-infusion of ILC2s reduces aGVHD and increases recipient survival. To evaluate the function of ex vivo ILC2s in GVHD biology, we expanded murine ILC2s as previously described (24) and verified their phenotype by flow cytometry (Supplemental Figure 2, A-C) and transcriptome analysis (Supplemental Figure 3, A and B, and Supplemental Table 1). Next, we performed survival studies in a murine model of allo-SCT with co-infused ILC2s. Splenic donor C57BL/6 (B6) T cells with a 1:1 ratio of ILC2s (BM, T cells + WT ILC2) or without ILC2s (BM + T cells) were infused into lethally irradiated haploidentical recipients on day 0. Co-transplantation of a single dose of ILC2s significantly improved survival, with $70 \%$ of recipients surviving 60 days after transplant (Figure 2A). There was a significant reduction in both the clinical score and weight loss in mice receiving ILC2s (Figure 2, B and C). On day 20, there was a decrease in pathology score in the colon and spleen (Figure 2D), with a nonsignificant decrease in the ileum and no difference in the lung and liver (Supplemental Figure 4A). Using a 2:1 ratio of ILC2s to T cells, we repeated the survival studies in a B6-into-BALB/c transplant model. There was a sig- 
A
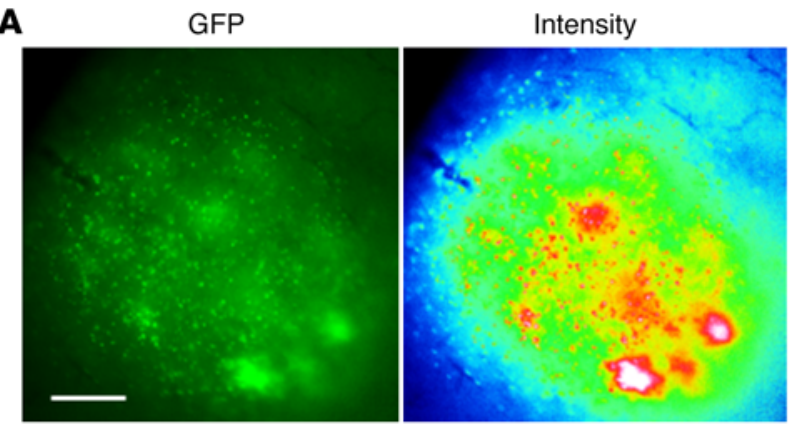

B
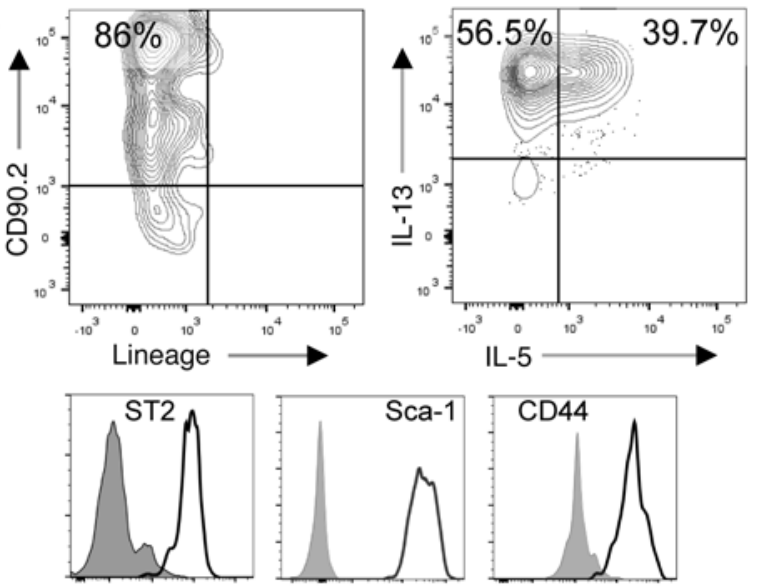

\section{C}
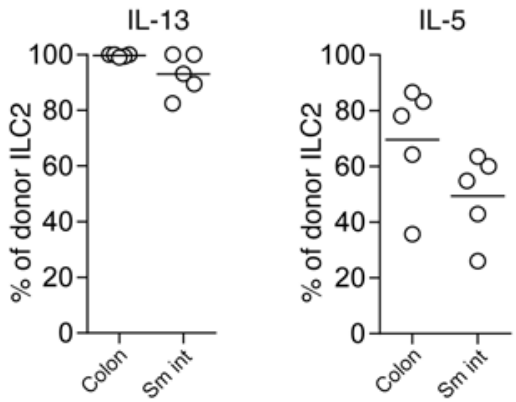

nificant improvement in survival (Figure 2E) and clinical GVHD score (Supplemental Figure 4B).

Treatment of a GVHD with ILC2s. While the use of ILC2s to prevent aGVHD would be beneficial, clinically it would be more impactful to use these cells to treat active aGVHD. ILC2s were infused 7 days after transplant, a time when donor $\mathrm{T}$ cells have initiated inflammation in GVHD target organs. In B6D2 recipients, infusion of a single dose of ILC2s significantly improved survival compared with no treatment (Figure 2F). In addition, there was a significant reduction in clinical GVHD score in recipients of ILC2s on day 7 (Figure 2G). We repeated our day 7 treatment experiments in the complete major histocompatibility complex (MHC) mismatch model and found significantly improved survival with ILC2 infusion (Supplemental Figure 4C). These findings indicate that a single infusion of ILC2s can significantly improve survival after the onset of GVHD.

ILC2s migrate to the GI tract and produce Th2-inducing cytokines. We were interested in determining whether ILC2s migrated to GVHD target organs after allo-SCT. For this, we infused B6-GFP' ILC2s into lethally irradiated B6D2 mice. While on day 12 after BMT only limited GFP signal was found in the LP, we observed
Figure 3. ILC2 evaluation in tissues after transplant. (A) Fluorescence microscopy of B6-GFP ILC2s in PPs 12 days after BMT; GFP imaging (left) and signal intensity (right). Magnification, $\times 40$; exposure, $200 \mathrm{~ms}$. Data represent 3 experiments; $n=6$ each. (B) Flow cytometry plots of B6-GFP ILC2 phenotype in the LP of BMT recipients 12 days after transplant, gated first as GFP+. (C) Percentage of B6-GFP ILC2s expressing IL-13 and IL-5; average \pm SEM. Results represent 3 independent experiments; $n=5$ each. Sm int, small intestine.

large clusters of $\mathrm{GFP}^{+}$ILC2s in Peyer's patches (PPs) of the colon and small bowel (Figure 3A). Evaluation of GFP ILC2s by flow cytometry confirmed that IL-33-activated cells had a surface phenotype similar to that of ex vivo expanded ILC2s (Figure 3B). Greater than $90 \%$ of donor $\mathrm{GFP}^{+}$ILCs in the GI tract expressed IL-13, while IL-5 expression varied, with more than $40 \%$ of ILC2s in the GI tract expressing IL-5 (Figure 3C). These findings indicate that ILC2s migrate to the GI tract, maintain their phenotype, and produce ILC2 signature cytokines.

Infused ILC2s reduce GI tract T cell burden and limit Th1 and Th17 induction. We evaluated the effect of the infusion of ILC2s on the generation of proinflammatory donor T cells in GVHD target organs. Co-infusion of ILC2s reduced the accumulation of donor $\mathrm{T}$ cells in PP, the colon, and small bowel, as determined by GFP intensity (Figure 4A). In addition, there was significantly less GFP in tissue homogenates (Figure 4B). To confirm these findings, we analyzed $\mathrm{T}$ cell infiltration into the lungs, liver, spleen, MLN, and colonic LP by flow cytometry (Figure 4C). Recipients of ILC2s had a reduction in donor lymphocytes in the LP of the colon (Figure $4 \mathrm{D}$ ), with a significant reduction in donor $\mathrm{CD} 8^{+} \mathrm{T}$ cells (Figure $4 \mathrm{E}$ ). There were significant reductions in the total number of LP CD4 ${ }^{+}$ and $\mathrm{CD}^{+} \mathrm{T}$ cells that expressed IFN $-\gamma$ and $\mathrm{CD} 4^{+} \mathrm{T}$ cells that generated IL-17A (Figure 4F). Infusion of ILC2s did not lead to a significant difference in the quantity of IFN- $\gamma$-producing donor $\mathrm{CD} 4^{+}$or $\mathrm{CD} 8^{+} \mathrm{T}$ cells in other GVHD target organs (Supplemental Figure 5, $\mathrm{A}$ and B). The decrease in IFN- $\gamma$-producing $\mathrm{CD}^{+}$and $\mathrm{CD} 8^{+} \mathrm{T}$ cells in the colon was not associated with an increase in $\mathrm{T}$ cells generating IL-10 or an increase in FoxP3-expressing Tregs (Supplemental Figure 5, C and D). These data indicate that co-infusion of ILC2s is associated with a reduction in the number of proinflammatory donor T cells generating IFN- $\gamma$ and/or IL-17A in the GI tract.

ILC2 suppression of a GVHD is independent of increased Th2 differentiation. Conceivably, ILC2s could induce donor Th2 polarization, which would mitigate aGVHD (25). To assess for Th2 cells after ILC2 infusion, we analyzed IL-4 production by donor $\mathrm{T}$ cells (Supplemental Figure 5E). We did not observe an increase in IL-4-producing donor T cells in recipients of ILC2s in the colon (Supplemental Figure 5F). To verify that the mechanisms behind ILC2 suppression of aGVHD are independent of the generation of $\mathrm{Th} 2$ cells, we performed survival studies using T cells from Stat $6^{-1-}$ mice (26). Recipients of Stat $6^{-1-} \mathrm{T}$ cells alone developed aGVHD, with all recipients dying before day 30 ; however, $80 \%$ of recipients given Stat $6^{-1-} \mathrm{T}$ cells with ILC2s survived long-term, with significantly reduced clinical scores by day 20 after BMT (Supplemental Figure 5, G and H). The ability of ILC2s to suppress aGVHD does not require the generation of donor Th2 cells.

ILC2 infusion enhances LP myeloid cells. We observed an increase in donor myeloid cells in the lower GI tract in recipients 
A

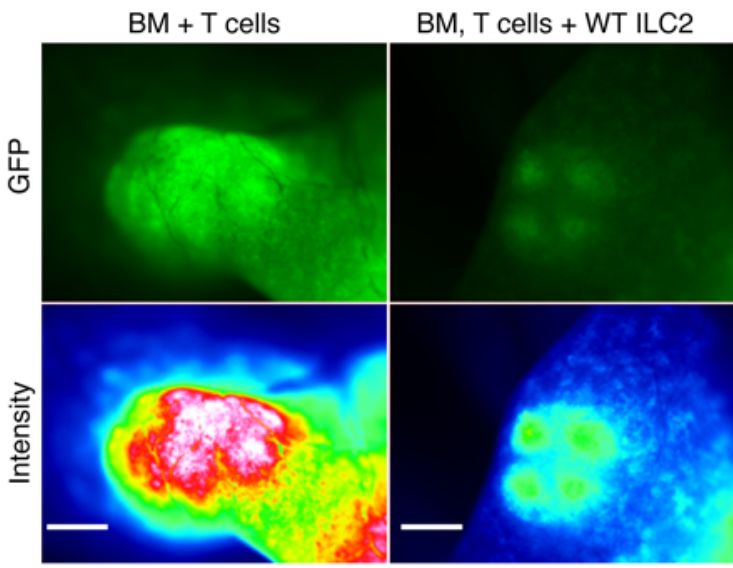

B

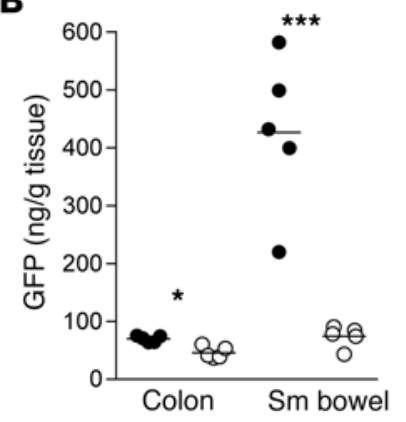

C

$\mathrm{BM}+$ $\mathrm{T}$ cells
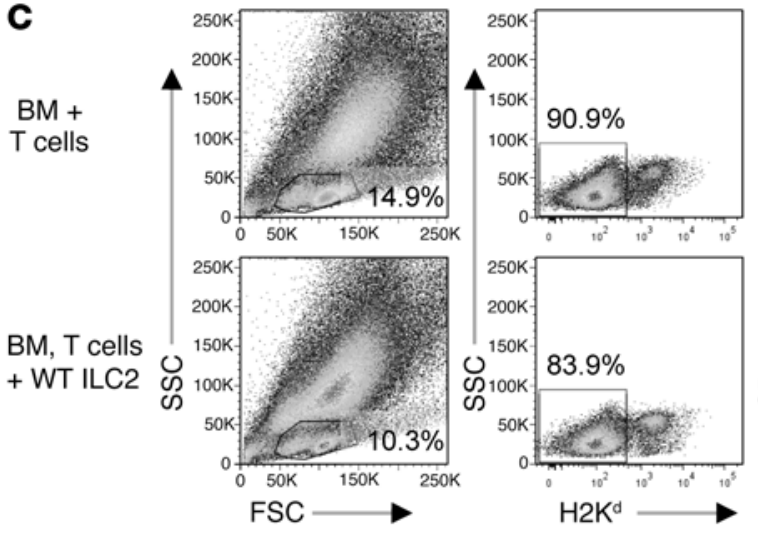

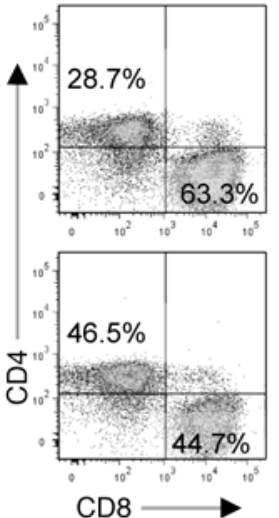

$\mathbf{F}$

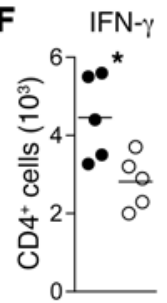

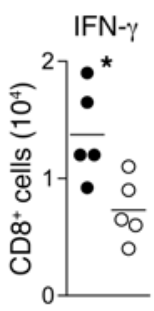

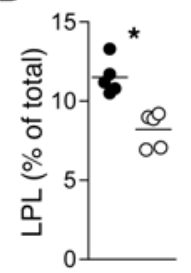

E

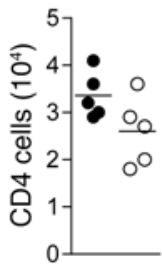

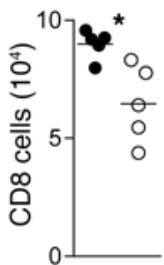

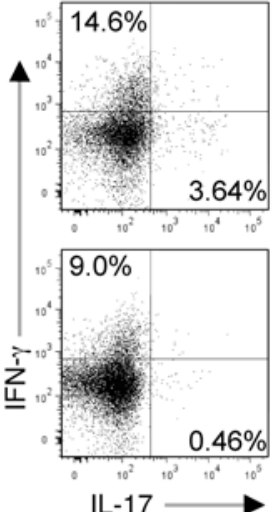

IL-17A

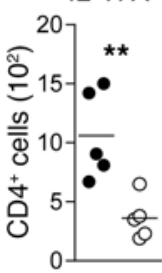

Figure 4. Co-transplantation of WT ILC2s reduces proinflammatory donor $\mathrm{T}$ cells numbers in the $\mathrm{Gl}$ tract. Donor $\mathrm{T}$ cells were evaluated in the $\mathrm{GI}$ tract 12 days after transplant using $\mathrm{GFP}^{+}$splenic $\mathrm{T}$ cells alone (BM + T cells) or GFP' $T$ cells and WT ILC2s (BM, T cells + WT ILC2). (A) Fluorescence microscopy of B6-GFP donor $T$ cells in PPs; GFP imaging (top row) and signal intensity (bottom row). Magnification, $\times 40$; exposure, 200 ms. (B) ELISA quantification of GFP in tissue homogenates from $B M+$ $\mathrm{CFP}^{+} \mathrm{T}$ cells (filled circles) and BM, GFP $^{+}$T cells + WT ILC2 (open circles) normalized to grams of tissue. Results represent 3 experiments ( $n=5$ each). Student's $t$ test with Welch's correction, ${ }^{*} P<0.05$, ${ }^{* * *} P<0.001$. Donor T cells from BMT were evaluated by flow cytometry 12 days after BMT. (C) Density plots and gating scheme for the evaluation of donor T cells and intracellular cytokine expression from colon. (D) Percentage of donor T cells in the LP $\mathrm{BM}+\mathrm{T}$ cells (filled circles) and BM, T cells + WT ILC2 (open circles). (E) The total number of $\mathrm{CD}^{+}$and $\mathrm{CD} 8^{+}$donor $T$ cells in the LP. (F) Total number of IFN- $\gamma$-producing donor $\mathrm{CD} 4^{+}$and $\mathrm{CD} 8^{+}$ $T$ cells and the number of donor $\mathrm{CD} 4^{+}$ T cells producing IL-17A in the LP. Data represent 2 independent experiments $(n=5)$. Student's $t$ test with Welch's correction, ${ }^{*} P<0.05,{ }^{* *} P<0.01$. of WT ILC2s (Supplemental Figure 6, A and B). We analyzed donor myeloid cells for expression of CD45, CD11b, Gr-1, Ly-6C, and Ly-6G, a phenotype that is consistent with MDSCs, which have been shown to suppress aGVHD (Figure 5A) $(12,27)$. We found a significant increase in donor MDSCs in the LP of colon and small bowel in ILC2-treated recipient mice (Figure 5B). However, MDSCs were not increased in the lung or liver of recipients of ILC2s (Supplemental Figure 6C). To test whether the function of ILC2s was dependent on MDSCs, we depleted myeloid cells using an anti-Gr-1 mAb in transplanted mice receiving either BM and $\mathrm{T}$ cells with or without donor ILC2s. Depletion of Gr-1+ ${ }^{+}$cells reduced the median survival of control $\mathrm{BM}$ and $\mathrm{T}$ cell recipients from 25 to 20 days (Figure 2A compared with Figure 5C). In contrast, depletion of $\mathrm{Gr}^{-1} 1^{+}$cells completely ablated the beneficial effect of ILC2s, with no difference being observed in survival between mice receiving donor $\mathrm{T}$ cells with anti-Gr-1 antibody and donor ILC2s with T cells plus anti-Gr-1 antibody (Figure $5 \mathrm{C}$ ). The ability of anti-Gr-1 mAb to abrogate the beneficial effects of donor ILC2s was dependent on the ability of ILC2s to prevent aGVHD, as infu- sion of anti-Gr-1 mAb had no impact on the outcome of recipient mice receiving only donor bone marrow cells (Figure 5C). There was a similar loss of efficacy of ILC2 infusion after depletion of Ly-6C ${ }^{+}$cells (Supplemental Figure 6D). Thus, the ability of ILC2s to prevent aGVHD is dependent on the presence of $\mathrm{Gr}-1^{+}$cells.

$I L-13$ is required for ILC2-induced donor MDSC suppression of inflammatory $T$ cells. Next, we performed survival studies to test the ability of Il13-/- ILC2s to suppress aGVHD (12). Compared with the infusion of $\mathrm{BM}+\mathrm{T}$ cells alone, there was no significant improvement in median survival for mice receiving BM, T cells, and $\mathrm{Il}_{133^{-1-}}$ ILC2s (Figure 6A). When compared with mice that received WT ILC2 treatment, only $25 \%$ of $I l 13^{--}$ILC2 recipients survived longterm as compared with $75 \%$ survival when WT ILC2s were given (Figure 6A). To further investigate the role of ILC2-derived IL-13 suppression of aGVHD, we evaluated IFN- $\gamma$ and IL-17A expression by donor $\mathrm{CD}^{+}$and $\mathrm{CD}^{+} \mathrm{T}$ cells in the colon. The decrease in $\mathrm{T}$ cells generating IFN- $\gamma$ and/or IL-17A observed in mice receiving WT ILC2s was lost after the infusion of Il13-- ILC2s (Figure 6B compared with Figure $4 \mathrm{~F}$ ). In addition, Il13 ${ }^{-}$ILC2s failed to 

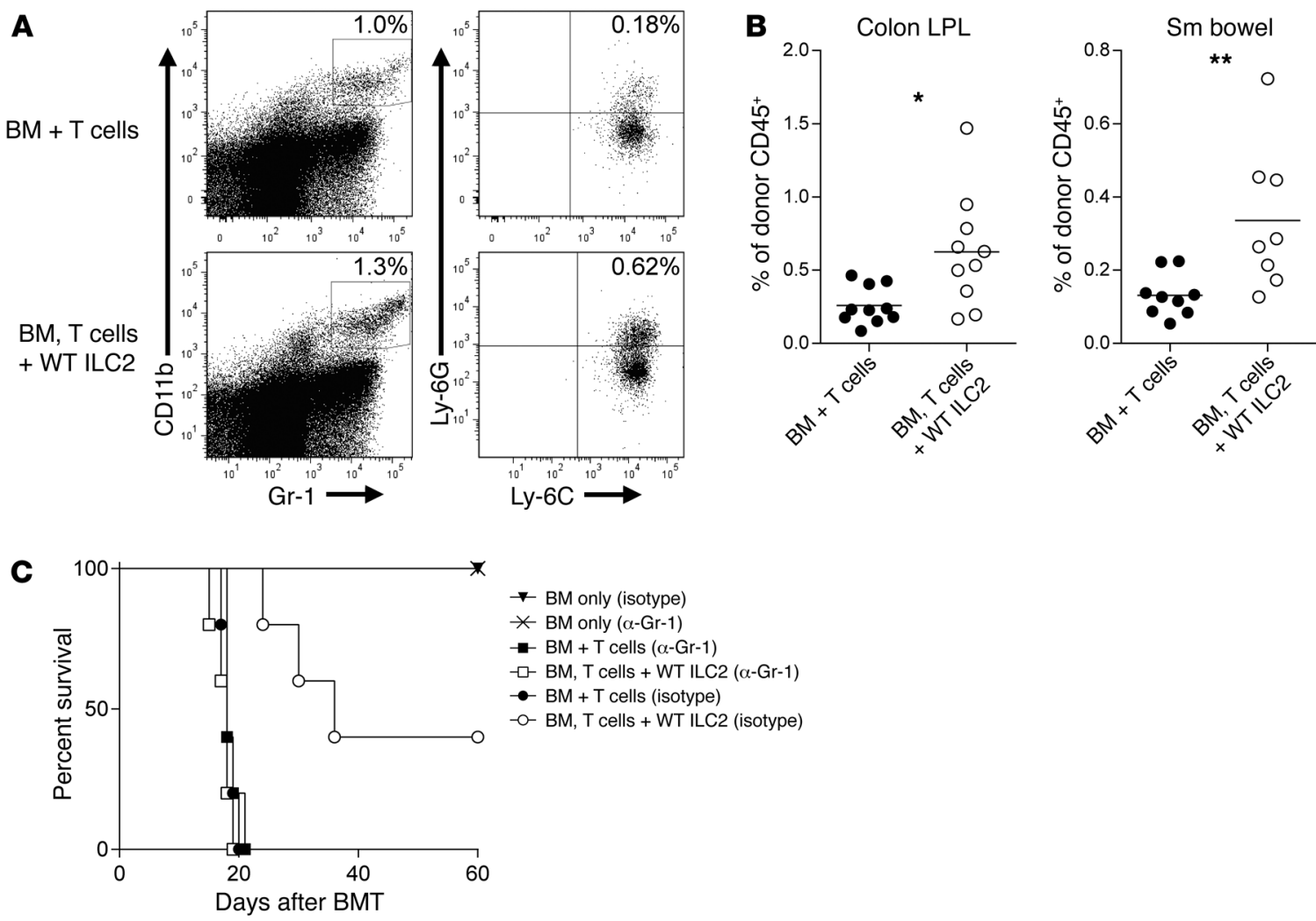

$\checkmark$ BM only (isotype)

* BM only $(\alpha-\mathrm{Gr}-1)$

- BM + T cells $(\alpha-\mathrm{Gr}-1)$

$\square$ BM, T cells + WT ILC2 $(\alpha-$ Gr-1 $)$

- BM + T cells (isotype)

o- BM, T cells + WT ILC2 (isotype)

Figure 5. Co-transplantation of WT ILC2s increases MDSC numbers in the GI tract. (A) Representative density plots of CD11 b+ Gr-1+Ly-6C+Ly-6C+ $M D S C s$ (gated on donor CD45+cells) 12 days after BMT. (B) Frequencies of MDSCs as a percentage of CD45+ granulocytes in the colon and small (Sm) bowel of BMT recipients 12 days after transplant. Results represent 2 independent experiments; bar graphs are average \pm SEM. Student's $t$ test with Welch's correction, ${ }^{*} P<0.05$, ${ }^{* *} P<0.01$. (C) Kaplan-Meier plot showing results of Gr-1 depletion. Lethally irradiated B6D2 mice (950 cGy) received $3.0 \times 10^{6} \mathrm{TCD} B M(B M$ only), BM plus $4.0 \times 10^{6}$ total splenic T cells (BM + T cells), or BM plus T cells with $4.0 \times 10^{6} \mathrm{IL}-33$-activated ILC2s (BM, T cells + WT ILC2). One group additionally received $200 \mu \mathrm{g}$ anti-Gr-1 ( $\alpha-G r-1)$, with another group receiving isotype control antibody twice weekly beginning 7 days after transplant. Results of 1 representative of 2 independent experiments are shown; $n=6$ per group.

enhance donor MDSCs in GVHD target organs (Figure 6C and Supplemental Figure 6E). These data indicate a critical role for IL-13 production by ILC2 cells to suppress donor T cell production of the proinflammatory cytokines IFN- $\gamma$ and IL-17A.

To evaluate how ILC2s impact the presence of MDSCs, we cocultured expanded ILC2s with BM-derived MDSCs (BM-MDSC) and quantified MDSCs 72 hours later. BM-MDSCs cultured with congenic ILC2s showed significantly enhanced survival compared with MDSCs cultured alone, MDSCs cultured in the presence of IL-13, and those cultured with IL-7 and IL-33 (Figure 6D). However there were a greater number of BM-MDSCs when cultured with wild type ILC2s than those cultured with IL-13-deficient ILC2s. To evaluate whether ILC2s and MDSCs require cell-to-cell contact, we cultured both populations in plates across a semipermeable membrane. Enhanced survival of MDSCs by ILC2s was abrogated in the absence of cell-to-cell contact (Figure 6D).

ILC2 infusions affect lower GI tract permeability. Previous investigators have demonstrated a critical role for ILC2s in the maintenance of epithelial integrity (13). Six days after BMT, we found there was no difference in intestinal permeability when comparing non-irradiated age-matched mice with BMT recipients of BM only; BM and T cells; or BM + T cells + WT ILC2s (Figure 7A). On days 12 and 20 after transplant, recipients of donor $\mathrm{T}$ cells had significantly enhanced epithelial permeability compared with healthy B6D2 mice as well as recipients of BM only (Figure 7, B and C). ILC2-treated mice had significantly improved epithelial integrity compared with control mice and showed no statistical difference compared with untreated $\mathrm{B} 6 \mathrm{D} 2$ or recipients of bone marrow alone (Figure 7, B and C). ILC2 infusion is associated with improved intestinal barrier function in the setting of reduced lower GI tract aGVHD.

Activated ILC2s produce amphiregulin in response to IL-33 (Supplemental Table 1), which promotes wound healing and epithelial barrier repair (15). We evaluated the ability of amphiregulin-deficient ILC2s ( $\mathrm{Areg}^{-1}$ ILC2) to protect against aGVHD mortality. Infusion of Areg-- ILC2 cells with donor T cells and BM led to diminished overall survival compared with recipients of WT ILC2 cells (Figure 7D and Figure 2A). However, the kinetics of the response differed from that found using Il13 ${ }^{-1-}$ ILC2 cells, with mortality occurring later after transplant (Figure 7D and Figure 6A). The infusion of $\mathrm{Areg}^{-1}$ ILC2s failed to increase epithelial barrier integrity (Figure 7E). Thus, the generation of amphiregulin by ILC2s is critical to the maintenance of the epithelial barrier in the GI tract mediated in part by ILC2s.

ILC2 cells do not inhibit the graft-versus-leukemia response. The antitumor response mounted by donor $\mathrm{T}$ cells is key to the clinical 
A
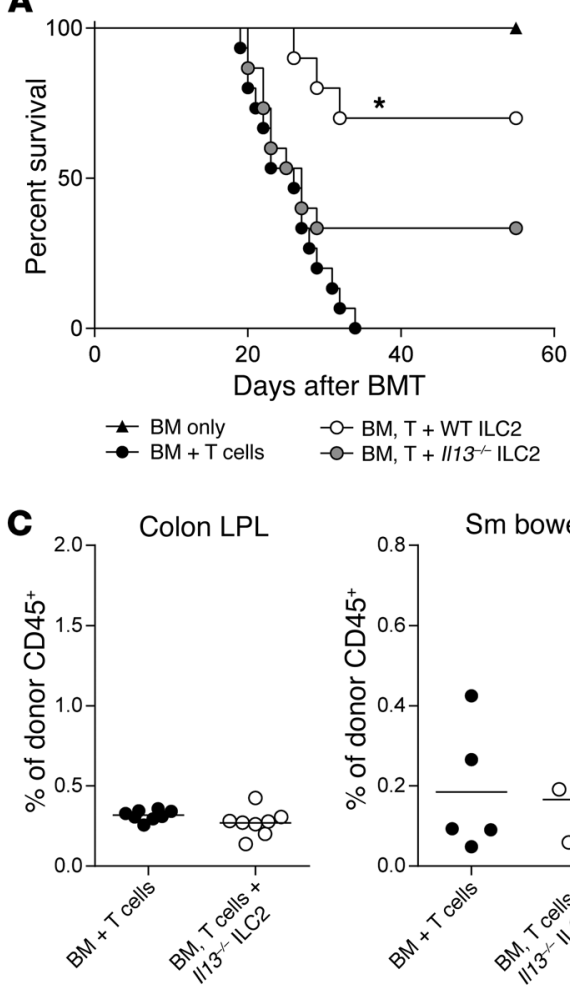

B

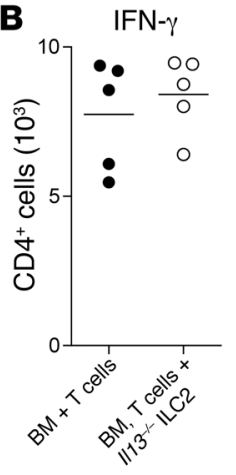

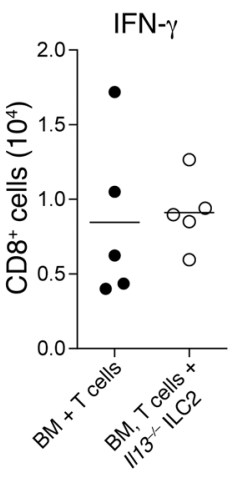

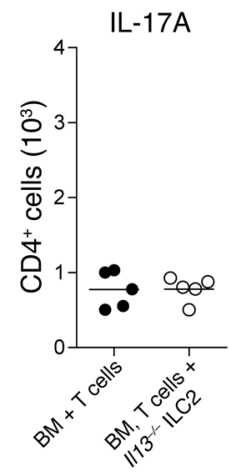

extensive tumor growth in the spleen, MLN, and inguinal lymph nodes. Mice that received $\mathrm{T}$ cells efficiently eliminated tumor cells when ILC2s were given (Figure 8A). In agreement with a previous report (30), infusion of a 1:1 ratio of Tregs to splenic $\mathrm{T}$ cells led to suppression of aGVHD, but with the loss of the graft-versus-tumor effect critical for allo-SCT (Figure $8 \mathrm{~B})$. When we evaluated a lower dose of Tregs (1:4 ratio), we found that the GVL response was preserved but that the majority of mice succumbed to GVHD (Figure 8B). These findings indicate that the survival of B6D2 recipient mice transplanted with $\mathrm{B} 6$ donor $\mathrm{T}$ cells and P815 tumor cells is significantly improved with co-administration of ILC2 cells compared with a $1: 1$ or 1:4 ratio of conventional $\mathrm{T}$ cells (Tcons) to Tregs (Figure 8C).

When given as treatment, MDSCs fail to suppress aGVHD (27). ILC2s and MDSCs were given on day 7 following allo-SCT in mice that received $\mathrm{BM}$ and splenic $T$ cells. When used therapeutically, MDSCs did not improve survival or clinical score compared with mice receiving donor $\mathrm{T}$ cells alone (Figure 8, $\mathrm{D}$ and $\mathrm{E})$. However, there was a substantial improvement in

efficacy of allo-SCT. We tested the graft-versus-leukemia (GVL) response by giving GFP-expressing blast crisis chronic myelogenous leukemia (BC-CML) tumor cells to allogeneic BMT recipients (28). Recipients of donor $\mathrm{T}$ cells had significantly fewer tumor cells in the spleen compared with mice receiving $\mathrm{T}$ cell-depleted (TCD) bone marrow alone, and this effect was independent of the infusion of donor ILC2s (Figure 8A).

Comparison of ILC2 with other cell therapy treatments for GVHD. Multiple cell populations have been generated in an attempt to mitigate the incidence and/or severity of aGVHD. Two populations that have recently been evaluated by our group are natural Tregs and IL-13 MDSCs $(12,29)$. To compare the function of ILC2s with these other immunosuppressive cell populations, we infused Tregs or ILC2s with donor T cells, TCD bone marrow, and luciferase-expressing P815 (luc-P815) tumor cells. Mice that received BM and luc-P815 without T cells showed the survival of mice receiving donor ILC2s, with a significant reduction in clinical score (Figure 8, D and E). Thus, ILC2s in vivo do not inhibit the GVL response found after the infusion of Tregs and have significantly improved function to treat aGVHD compared with MDSCs.

Human ILC2 expansion. While these findings indicate a critical role for the administration of donor ILC2s to control lower GI tract GVHD, for clinical translation, we needed to expand human ILC2s (hILC2s) ex vivo. We isolated CD34+ cord blood cells and cultured these cells with IL-33 and IL-2 with/without IL-25. Culture with IL-2/IL-33 alone led to 2.5- to 3-log expansion of cells; however, the addition of IL-25 allowed for the persistent expansion of cells through day 21. Within this bulk population, $\mathrm{CRTH}_{2} \mathrm{CD}_{161} 1^{+}$lineage-negative hILC2s were found (Figure $8 \mathrm{~F}$ and Supplemental Figure 6F). Thus, human ILC2 cells can be expanded ex vivo from $\mathrm{CD} 34^{+}$cord blood progenitors. 
A

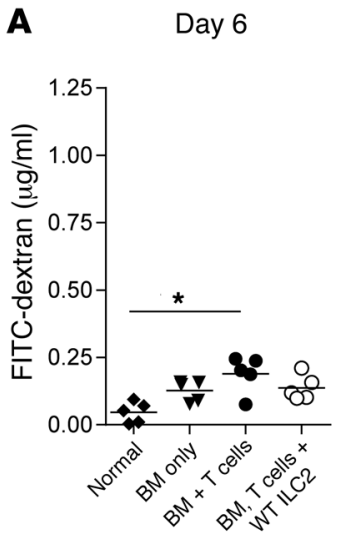

B

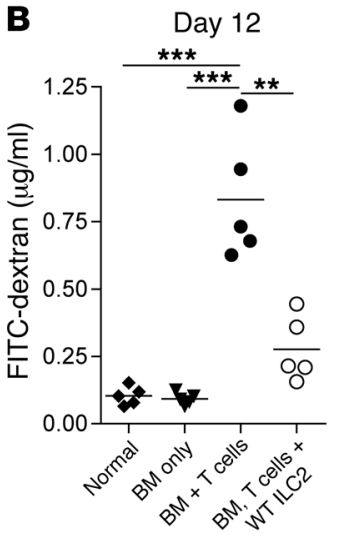

D

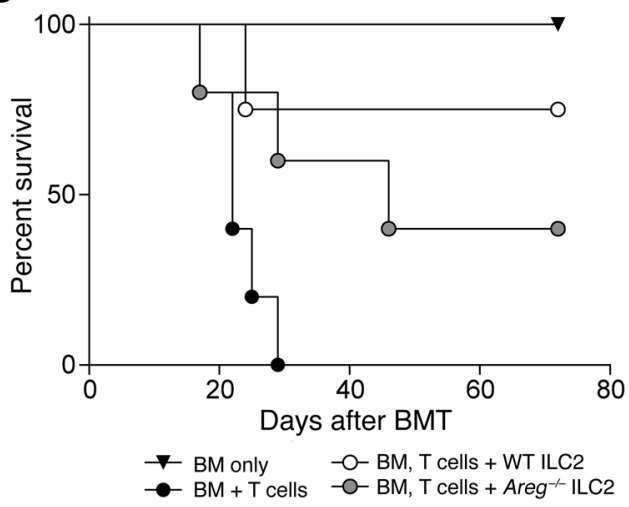

C

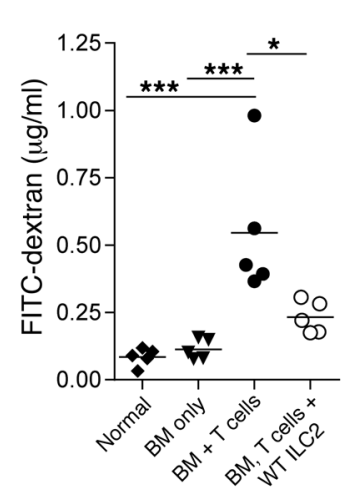

E

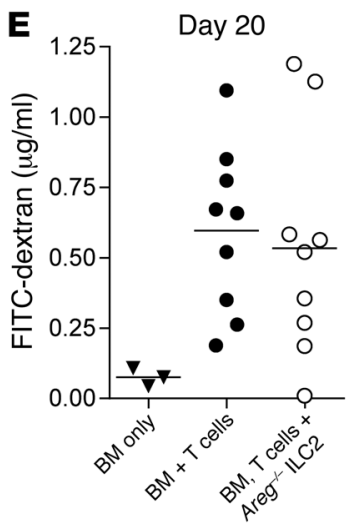

Figure 7. ILC2 treatment improves intestinal barrier function and does not abrogate the GVL response. Quantification of FITC-dextran in the serum of BMT recipients (A) 6 days, (B) 12 days, and (C) 20 days after transplant. One representative of 2 independent experiments shown; mean \pm SEM ( $n=5$ per group). Statistical analysis by 1-way ANOVA with Bonferroni's correction for multiple comparisons, ${ }^{*} P<0.05,{ }^{* *} P<0.01$, ${ }^{* *} P<0.001$. (D) Kaplan-Meier plot of B6D2 recipients of B6 TCD BM (BM only), BM plus splenic T cells (BM + T cells), or BM plus T cells with cultured Areg $^{-/-}$ILC2s (BM, T cell + Areg $^{-1-}$ ILC2) were evaluated for survival following allo-SCT. One representative of 2 experiments shown ( $n=5$ per group in each experiment). WT ILC2 group shown represents one experiments from Figure 2A. (E) Quantification of FITC-dextran in the serum of BMT recipients 20 days after transplant. One combined representative of 2 independent experiments is shown; mean \pm SEM ( $n=5$ per group).

\section{Discussion}

Steroid-refractory aGVHD of the lower GI tract is the most common cause of morbidity and mortality in patients who develop aGVHD after stem cell transplantation. Here, we demonstrate that the administration of donor ILC2s at the time of allo-SCT and up to 1 week after transplant significantly improved survival and clinical GVHD scores. ILC2s did not abrogate the antitumor GVL response, which may not be surprising given their primary function in the lower GI tract, which is not the site of tumor growth in our models or for patients undergoing allo-SCT. Thus, ILC2 infusion provides, what we believe to be, a novel approach to therapy for lower GI tract GVHD.

One complication of allo-SCT is the incomplete reconstitution of immune cells from donor bone marrow. Some cell populations, such as B-1 lymphocytes, are generated preferentially from fetal liver and/or bone marrow and not adult bone marrow cells (31). In contrast, ILC2s in the GI tract, which have been shown to derive from adult bone marrow precursors, have extremely slow reconstitution kinetics after BMT. We find significantly decreased numbers of both donor and host ILC2s, with a greater decrease on day 28 compared with day 1 after allo-SCT. These findings support clinical data regarding the paucity of circulating ILC2s 12 weeks after allogeneic stem cell transplantation (32). There are at least two potential hypotheses for the impaired recovery of ILC2s from donor bone marrow after allogeneic BMT. First, despite their generation from bone marrow cells during development, precursor ILC2s may not be effectively generated from adult bone marrow precursor cells. In support of this hypothesis, a recent publica- tion demonstrated poor recovery in patients with the common $\gamma$-chain deficiency or JAK3 mutation of ILCs, other than ILC1s, after stem cell transplantation. Interestingly, the impaired recovery of ILCs after stem cell transplantation was much greater in patients receiving non-myeloablative conditioning therapy. The impaired recovery of ILCs after transplant was not associated with an increased risk of infectious diseases, suggesting that ILCs may not be critical in the presence of $\mathrm{T}$ and $\mathrm{B}$ cells for the control of infectious pathogens (33). Our findings suggest that ILCs may only be critical in specific uncommon settings, such as assisting in the control of the persistent inflammation in the GI tract during severe aGVHD. Thus, a substantial number of patients after allogeneic SCT may function normally in the absence of a sufficient number of ILC2s. A second hypothesis is that precursor ILC2s are present in the donor bone marrow population but that cellular plasticity and the significant proinflammatory environment after allogeneic BMT in the lower GI tract converts these precursor ILC2s to ILC1s and/or ILC3s (34-36). Both of these mechanisms could contribute to the poor recovery of ILC2s in mice after allogeneic BMT.

ILC2s have been found to impact the presence of alternatively activated macrophages (37). To the best of our knowledge, our group is the first to demonstrate a role for ILC2s in the presence of MDSCs, which required ILC2 production of IL-13. Previous studies have shown that IL-13 is capable of activating MDSCs through activation of STAT1 and STAT6, and this leads to upregulation of arginase and INOS, which are critical to the immunosuppressive function of MDSCs $(38,39)$. Stromal cell production of IL-13 has been demonstrated to be important in this process (40). Here we 
A

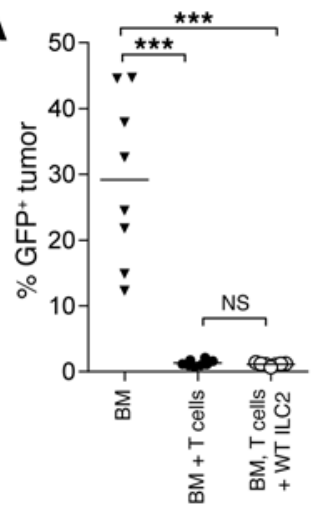

Figure 8. Comparison of ILC2s and other cellular therapies for GVHD. (A) Percentage of GFP+ BC-CML tumor in host spleen 21 days after BMT in the B6-into-B6D2 model. Results represent 2 independent experiments $(n=8$ each). Analysis by Student's $t$ test with Welch's correction, ${ }^{* *} P<0.001$. (B) IVIS imaging of luc-P815 tumor in B6D2 BMT recipients 15 days after transplant comparing recipients of BM and luc-P815 cells (BM only); BM with splenic T cells and luc-P815 cells (BM + T cells); BM, splenic T cells, Tregs, and luc-P815 cells (BM, T cells + Treg); and BM, splenic T cells, WT ILC2s, and luc-P815 cells (BM, T cells + WT ILC2). Results represent 2 independent experiments; $n=5$ in each group. (C) KaplanMeier plot comparing the survival of the allo-SCT recipients described in B. Log-rank (Mantel-Cox) test, ${ }^{* *} P<0.01$. (D) Kaplan-Meier plot comparing the survival of recipients of allo-SCT (B6 into B6D2 model) that received BM alone (BM only); BM and splenic T cells (BM + T cells); and those that received BM and splenic $T$ cells at the time of transplant with either WT ILC2s (BM, T cells + WT ILC2) or BM-MDSCs (BM, T cells + MDSC) 7 days after BMT. Results represent 2 combined experiments ( $n=10$ or more per group). Log-rank (Mantel-Cox) test. (E) Clinical scores of recipients from the survival study in $\mathbf{D}$. ${ }^{* *} P<0.01$ by 2 -way ANOVA, with Bonferroni correction for repeated measures of multiple comparisons. (F) Expansion of human ILC2s (hILC2) from SR-1-stimulated CD34+ cord blood cells in the indicated cytokines. hILC2s were enumerated as CRTH2+CD161- Lin- cells (Supplemental Figure 6F) from days 3 to $21 ; n=3$.
B

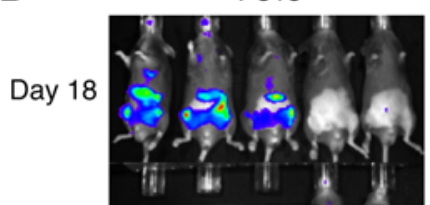

Day 22

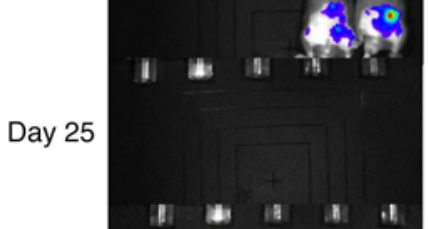

Day 29

Day 32
P815 + T cells

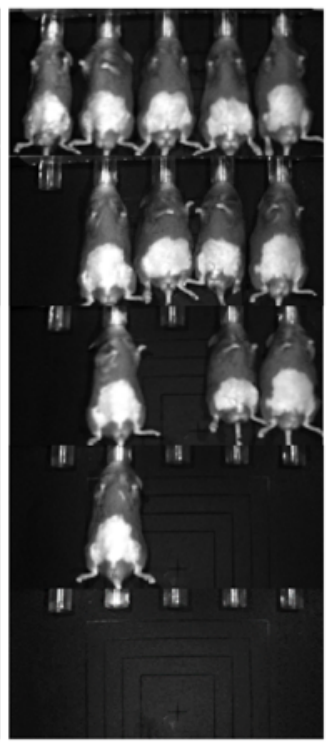

P815, T cells + WT ILC2

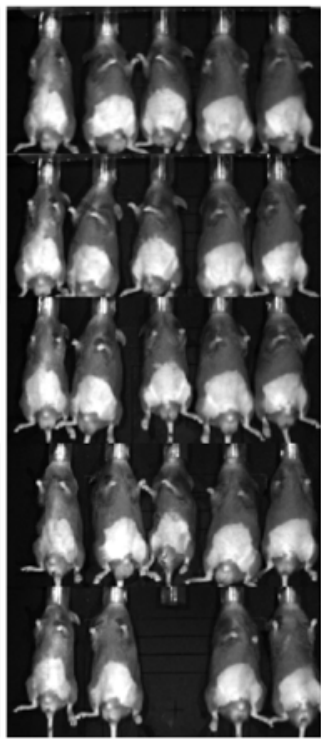

P815, T cells + Tregs $\left(4.0 \times 10^{6}\right)$

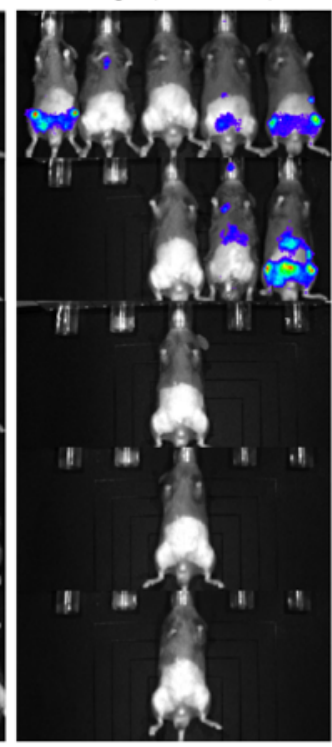

P815, T cells + Tregs $\left(1.0 \times 10^{6}\right)$

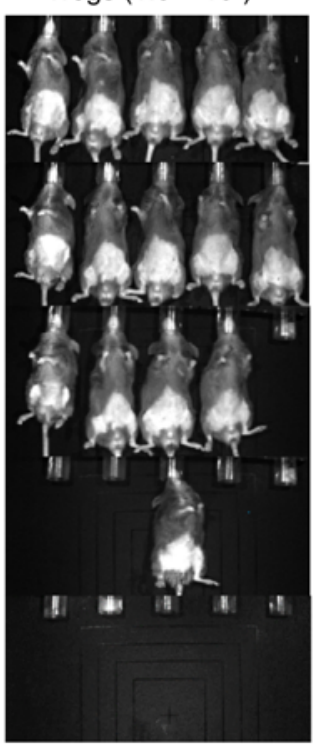

\section{c}

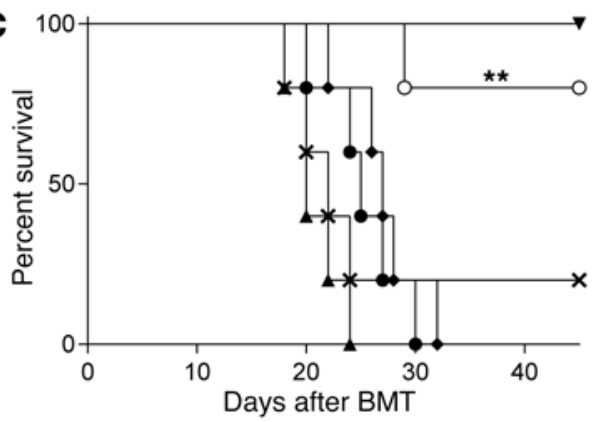

$\rightarrow$ BM only

^- P815

- P815 + T cells

- - P815, T cells + WT ILC2

$-x$ P815, T cells + Treg $\left(4.0 \times 10^{6}\right)$

$\leftarrow$ P815, T cells + Treg $\left(1.0 \times 10^{6}\right)$

D

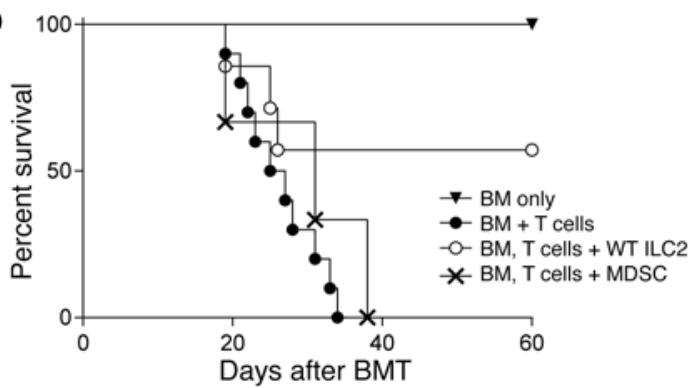

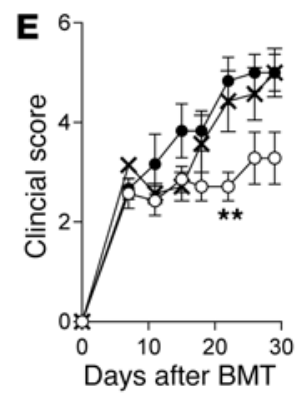

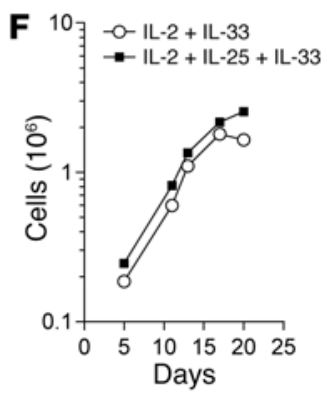


demonstrate that ILC2s can maintain the viability of MDSCs in vitro. This process requires IL-13 generated by ILC2s and cell-tocell contact between MDSCs and ILC2s. How this functions in vivo is a current focus of our research.

IL-33, which is critical for the generation of ILC2s, is a ligand for the IL-33 receptor also termed ST2. Previous work evaluating biomarkers as predictors of outcome for patients undergoing allogeneic stem cell transplantation has found that elevation of ST2 was associated with a lack of therapeutic response for GVHD on day 28 after transplant and increased mortality 6 months after transplantation (41). In those evaluations, ST2 levels were greater for patients undergoing myeloablative compared with reduced-intensity conditioning regimens. These data were confirmed in a study of recipients of cord blood transplant (42). We have shown that increased generation of IL-33 after transplantation is associated with increased severity of GVHD in murine models (43), and our group and another have found that blocking the interaction of IL-33 with ST2 diminished aGVHD (44). We hypothesize that the increased levels of ST2 after transplant are, in part, due to the loss of ILC2s in the GI tract that would respond to IL-33. Persistent inflammation in the GI tract would lead to increased production of soluble ST2, which would be greater after myeloablative conditioning therapy due to the greater loss of ILC2s from the increased dose of chemotherapy or irradiation. Our data would suggest that infusion of donor ILC2s, by markedly decreasing the accumulation of IFN- $\gamma$ - and IL-17-producing T cells in the GI tract, diminishes this proinflammatory environment. We hypothesize that ST2 levels would significantly decrease after ILC2 infusion, which is currently being evaluated.

AREG is a member of the epithelial growth factor receptor family and plays a critical role in wound healing after injury (45). Basophils, mast cells, $\mathrm{CD}^{+}{ }^{+} \mathrm{T}$ cells, eosinophils, and dendritic cells in addition to ILC2s can generate AREG. In some specific instances, FoxP3 ${ }^{+}$Tregs that express ST2 can generate AREG (46). Our data are consistent with a critical role for the production of AREG by ILC2s in their function to promote lower GI tract barrier function. However, we did not determine the relative contribution of ILC2s to the generation of AREG in the lower GI tract after transplant. Future work is required to determine whether AREG production by these other immune cells (and by non-immune cells) is critical to promoting GI tract epithelial integrity after the development of acute GVHD.

There are several additional questions that remain to be addressed from our findings. The mechanism underlying the tissue-specific effects of conditioning therapy on ILC2s found in the lung compared with those in the GI tract is not clear. This could be related to differences in the microbiota at each site. Alterations in the microbiome after conditioning therapy could activate ILC2s in the GI tract, leading to enhanced depletion of cycling ILC2s after conditioning therapy. Furthermore, there are clear differences in the supporting immune and nonimmune cells at each site that also could play a role in the cell cycle status of ILC2s in the lung compared with the GI tract. Finally, how ILC2s and ILC3s interact during aGVHD is not clear. As our hypothesis focused on the effects of conditioning therapy on regulatory immune populations in the GI tract, and since ILC3s in the GI tract are not depleted by conditioning therapy, we have not performed evaluations focusing on these cells.
In conclusion, we demonstrate that restoration of ILC2s can treat GI tract aGVHD; is dependent on the generation of IL-13 and amphiregulin by the ILC2s; and unlike Tregs does not impact the GVL response. These findings provide a strong preclinical rationale for testing the infusion of ILC2 cells to treat patients with steroid-nonresponsive lower GI tract GVHD.

\section{Methods}

Mice. B6, BALB/cJ, B6.129S2 Stat6 ${ }^{-/}$(termed Stat6 $\left.6^{-/}\right)$, and C57BL/6J × $\mathrm{DBA} / 2 \mathrm{~F}_{1}$ (termed B6D2) mice were purchased from The Jackson Laboratory. The generation of enhanced GFP-expressing C57BL/6 mice has been described previously (47). C57BL/6 Il13-eGFP and C57BL/6 Areg/- mice have been described previously (24, 48). Donor and recipient mice were age-matched males between 8 and 16 weeks.

Isolation and expansion of murine ILC2s and MDSCs. Ten- to 12-week-old B6 mice were given $0.4 \mu \mathrm{g}$ recombinant mouse IL-17E/ IL-25 (R\&D Systems) by i.p. injection for 4 days. On day 5 , cells were isolated from the MLN and peritoneum by peritoneal lavage using RPMI with $10 \%$ fetal bovine serum (complete media). ILC2s were isolated by negative selection with a MACs column using biotinylated antibodies (anti-CD8 $\alpha$ [clone 53-6.7], anti-CD4 [RM 4.4], anti-CD3 $\varepsilon$ [clone 145-2C11], anti- $\gamma \delta$ TCR [UC7-13DS], anti-TER119 [TER-119], anti-B220 [RA3-6B2], anti-CD11b [M1/70], anti-NK1.1 [PK136], eBioscience; anti-CD11c [N418], anti-CD19 [MB19-1], anti-Ly6G [1A8], and anti-CD49b [DX5], BioLegend) and Streptavidin MicroBeads (Miltenyi 130-048-101). Cells were cultured for 6 days in complete media with rIL-7 and rIL-33 (10 ng/ml) (PeproTech), with the media changed every 2 days. ILC2 activation was evaluated using flow cytometry on day 6 by intracellular cytokine staining of IL-5 and IL-13. For BM-MDSCs, freshly isolated CD90-depleted BM was plated at $3.0 \times 10^{5}$ cells $/ \mathrm{ml}$ and incubated 4 days with $250 \mathrm{U} / \mathrm{ml}$ recombinant GM-CSF (catalog 315-03) and $100 \mathrm{ng} / \mathrm{ml} \mathrm{G-CSF}$ (catalog 250-05) (PeproTech). On day 4, $80 \mathrm{ng} / \mathrm{ml}$ recombinant IL-13 (catalog 210-13) (PeproTech) was added to the cultures. After 4 days, CD11b ${ }^{+}$cells were isolated by magnetic bead separation using the Miltenyi CD11b ${ }^{+}$selection kit (catalog 130-049-601) (Miltenyi Biotec). CD11 $\mathrm{b}^{+}$cells were analyzed for expression of Gr-1; approximately $96 \%$ of the live cells were CD11 $\mathrm{b}^{+} \mathrm{Gr}-1^{+}$. For ILC2/MDSC coculture experiments, ILC2s and MDSCs were plated at a 1:1 ratio at a density of $2.0 \times 10^{5}$ cells $/ \mathrm{ml}$, in conventional 24 -well plates or in $5.0-\mu \mathrm{m}$ filter Transwell plates, with either $10 \mathrm{ng} / \mathrm{ml} \mathrm{IL-7}$ and IL-33 or $80 \mathrm{ng} / \mathrm{ml}$ IL-13 for 3 days before quantitation of live MDSCs by flow cytometry.

RNA sequencing evaluations. RNA was extracted from cultured ILC2s using the RNEasy Mini Kit (QIAGEN), and mRNA sequencing (mRNAseq) libraries were prepared according to the TruSeq protocol (Illumina). For mRNA-seq, samples were processed as previously described by The Cancer Genome Atlas (49). Bases and quality control assessment of sequencing were generated by CASAVA 1.8. QC-passed reads were aligned to the murine reference genome using MapSplice (50). Aligned reads were sorted and indexed using SAMtools (http://www.htslib. org/), and then translated to transcriptome coordinates and filtered for large inserts and zero mapping quality using UBU v1.0 (https://github. $\mathrm{com} / \mathrm{mozack} / \mathrm{ubu}$ ). For the reference transcriptome, UCSC murine knownGenes (51) was used, with genes located on nonstandard chromosomes removed. The abundance of transcripts was then estimated using an expectation-maximization algorithm implemented in the software package RSEM (52) v1.1.13. Estimated counts were normalized to the upper quartile (53) prior to comparison of expression across proto- 
cols. Normalized counts were converted to expression rank values for comparison with microarray data. RNA-seq raw data files have been deposited in the NCBI's Gene Expression Omnibus (GEO GSE95811).

Comparison of ILC2 mRNA-seq with published microarray data. Murine ILC gene signatures and raw microarray data were extracted from Robinette et. al. and relevant NCBI GEO datasets (54). Microarray data were processed in $\mathrm{R}$ (http://www.R-project.org, R Development Core Team, Vienna, Austria) to determine transcript relative expression values using the Robust Multichip Average algorithm implemented in the oligo package (55). Transcript-level inference of relative expression was done by cross-referencing normalized probe signal values with the "mogene10sttranscriptcluster" database (Affymetrix). Normalized signal values were converted to expression rank values for comparison with mRNA-seq data. Spearman rank correlations between gene expression values of ILC-associated genes for measured ILC2s $(n=6)$ and published ILC1s ( $n=2,12$ comparisons), ILC2s ( $n=2,12$ comparisons), and ILC3s ( $n=8,48$ comparisons) were calculated and used as a statistic. Significance between sample groups was evaluated using the Kruskal-Wallis test. Mean rank expression values for each gene from each published group were plotted against mean rank expression values in the cultured ILC2s. Significance was tested using the "cor.test" module in R (method = "spearman").

Transplantation models. Total T cells were isolated using a Cedarlane $\mathrm{T}$ cell recovery column kit (Cedarlane Laboratories), followed by antibody depletion using PE-conjugated anti-mouse B220 (RA3-B62) and anti-mouse CD25 (3C7) antibodies (eBioscience) and magnetic bead selection using anti-PE beads (130-0480801, Miltenyi Biotec). TCD bone marrow was prepared as described previously (56). The day prior to transplantation, recipient mice received either $950 \mathrm{cGy}$ (B6D2) or $800 \mathrm{cGy}(\mathrm{BALB} / \mathrm{c})$ of total body irradiation. For the B6, Il13 ${ }^{-/}$, Areg - $^{--}$and Stat $^{-/-}$into B6D2 or B6 into BALB/c transplants, recipients were intravenously injected with either $4 \times 10^{6} \mathrm{~T}$ cells and 3 $\times 10^{6} \mathrm{TCD}$ BM cells, or $5 \times 10^{5}$ total T cells and $5 \times 10^{6} \mathrm{TCD}$ BM cells, respectively. For ILC2 treatment groups, B6D2 or BALB/c recipients also received $4 \times 10^{6}$ or $1 \times 10^{6} \mathrm{CD} 0^{+}$ILC2s, respectively. Recipients were monitored twice a week and scored for clinical GVHD symptoms (designated "clinical score") using a semiquantitative scoring system as previously described $(57,58)$; animals were coded for these evaluations. The sample size was chosen for the effect size needed based on our previous experience with sample sizes needed to demonstrate a significant difference in GVHD scoring between control and treated groups. Investigators were blinded to the clinical score and the pathological evaluations. For the scoring evaluation experiments, the inclusion of 9-12 recipients provided a power of $90 \%$ to detect a difference of 14 days in the median GVHD score of $\geq 5$ with an $\alpha$ error of $<0.05$ between control and treated groups. For all experiments, a control group received TCD bone marrow alone without additional $\mathrm{T}$ cells, which controlled for the presence of $\mathrm{T}$ cells in the marrow inoculum and potential infectious complications during aplasia. MDSC depletion was performed by i.p. injecting recipients twice weekly starting on day 7 with $200 \mu \mathrm{g}$ anti-Gr-1 antibody (RB6-8C5) or anti-Ly-6G antibody (Monts-1; BioXCell) until the termination of the experiment.

Evaluation of radiation and chemotherapy sensitivity. B6D2 mice received 950 cGy total body irradiation or cyclophosphamide (200 $\mathrm{mg} / \mathrm{kg}$ ) (Santa Cruz Biotechnology Inc., sc-219703) by i.p. injection. Twenty-four hours or 28 days later cells were isolated from the LP, MLN, and spleen for evaluation by flow cytometry. Quantitation of
ILCs was performed using the absolute event count of live cells gated as lineage negative and $\mathrm{CD} 9 \mathrm{O}^{+}$. Expression of ICOS or ROR $\gamma \mathrm{t}$ was used to enumerate ILC2s or ILC3s, respectively.

Fluorescence stereomicroscopy and GFP quantification. Organs from anesthetized animals were imaged with a Zeiss SteReo Lumar V12 microscope with GFP bandpass filter (Carl Zeiss MicroImaging Inc.) at room temperature. AxioVision (Carl Zeiss) software was used to determine GFP intensities. Control and treatment recipient organs were imaged using the identical magnification (mag) and exposure (exp) times on day +12 : PP were imaged at exp $200 \mathrm{~ms}$, mag $\times 40$. Organs from recipient animals were homogenized, and absolute GFP levels were determined by ELISA (Cell Biolabs, AKR-121). No GFP was found in mice receiving sham treatment. Detailed experimental procedures were conducted as described previously (56).

Cell isolation from GVHD target organs. Spleen, liver, lung, MLN, and colon were excised and weighed. LP lymphocytes were isolated using the Miltenyi LP dissociation kit (catalog 130-097-410) as per the manufacturer's instructions. Livers and lungs were digested in a solution of $1 \mathrm{mg} / \mathrm{ml}$ collagenase A (Roche) and 75 U DNase I (SigmaAldrich) in RPMI 1640/5\% newborn calf serum. Digested tissues were treated with ACK lysis buffer to remove rbc and were passed through 100- $\mu \mathrm{m}$-pore-size cell strainers. Leukocytes were collected at the interface of a $40 \% / 80 \%$ Percoll (Sigma-Aldrich) gradient in RPMI 1640/5\% newborn calf serum. The pelleted cells were washed in PBS/2\% FBS. Spleens and MLN were teased apart, treated with ACK lysis buffer, and washed in PBS/2\% FBS.

GVL analysis. BMTs were performed as described, and at the time of transplant B6D2 recipients were given $2.5 \times 10^{4} \mathrm{GFP}^{+} \mathrm{BC}-\mathrm{CML}$ or luciferase-expressing P815 (luc-P815 tumor cells, ATCC TIB-64) (Lentivirus BLIV101PA-1, System Biosciences). Recipients of BC-CML tumor were euthanized 21 days after BMT, and splenocytes were evaluated for GFP expression by flow cytometry. Tregs were isolated from B6 splenocytes using the $\mathrm{CD} 4{ }^{+} \mathrm{CD} 25^{+}$Regulatory $\mathrm{T}$ Cell Isolation Kit (130-091-041, Miltenyi Biotec). Recipients of luc-P815 were monitored for survival and tumor infiltration by luciferase imaging on the IVIS Kinetic Optical System. Mice received $3 \mathrm{mg}$ D-luciferin (PerkinElmer) 10 minutes prior to imaging. These cell lines were validated by MHC typing, GFP or luciferase expression, and growth parameters.

Flow cytometry analysis. ILC2s were evaluated by flow cytometry for linage markers (catalog 88-7772) and CD90 (HLS51), ICOS (7E.17G9), CD127 (A7R34), T1/ST2 (RMST2-2), Sca-1 (D7), CD44 (IM7), CD25 (PC61.5), and GITR (DTA-1); and intracellular staining for IL-4 (11B11), IL-13 (eBio13A), IL-5 (TRFK5), IFN- $\gamma$ (XMG1.2), and IL-22 (1H8PWSR). T cells were evaluated by surface staining of $\mathrm{H} 2 \mathrm{~K}^{\mathrm{d}}$ (34-1-2S), CD4 (GK1.5), CD8 (clone 53-6.7); and intracellular staining for IFN- $\gamma$ (XMG1.2), IL-17 (ebio17B7), IL-4 (11B11), IL-10 (JESS-16E3), and FoxP3 (FJK-16s). For analysis of MDSCs, donor cells were stained for the surface markers CD45 (30-F11), CD11b (M1/70), CD11c (N418), Gr-1 (RB6-8C5), Ly-6C (HK1.4), and Ly-6G (1A8) (eBioscience). Sample acquisition was performed using a BD FACSCanto or BD LSRFortessa (BD Bioscience).

FITC-dextran permeability assay. Intestinal permeability was assessed by luminal enteral administration of FITC-dextran 4000 (Sigma-Aldrich), a non-metabolizable macromolecule that is used as a permeability probe. Mice were fasted for 4 hours prior to gavage with FITC-dextran ( $40 \mathrm{mg} / 100 \mathrm{~g}$ body weight). After 4 hours, whole blood was collected by cardiac puncture, and FITC-dextran measurements were performed in triplicate by fluorimeter using a PerkinElmer 
EnSpire multimode reader. Dilutions of FITC-dextran in PBS were used as a standard curve, and absorption of $50 \mu$ lof serum or standard was measured at $488 \mathrm{~nm}$.

Expansion of human ILC2s. Isolated CD $34^{+}$cells from frozen umbilical cord blood cells (NHLBI Umbilical Cord Blood Unit) were expanded with $50 \mathrm{ng} / \mathrm{ml}$ IL-6, Flt3L, SCF, TPO (PeproTech) and $750 \mathrm{nM} \mathrm{SR} 1$ (Sigma-Aldrich) for 15 days. Cells were then cultured in 24-well plates at $0.5 \times 10^{6} / \mathrm{ml}$ with $100 \mathrm{U} / \mathrm{ml} \mathrm{IL-2,} 50 \mathrm{ng} / \mathrm{ml} \mathrm{IL-25}$, and $50 \mathrm{ng} / \mathrm{ml}$ IL-33 (PeproTech), with RPMI containing 10\% human antibody serum (Valley Biomedical) and 1\% penicillin and streptomycin (Thermo Fisher Scientific). Cytokines were replenished every 3 days. On days 5, $10,15,20$, and 25 , cells were harvested, and flow cytometry was performed. Human ILC2s were characterized as CRTH2+ (BM16) CD161 ${ }^{+}$ (HP-3G10) and T cell (OKT3), B cell (HIB19), monocyte (P67.6) lineagenegative cells. All antibodies were purchased from eBioscience.

Statistics. Survival differences were evaluated using a Mantel-Cox log-rank test. Survival curves were generated using the Kaplan-Meier method. Differences in GVHD clinical and pathology scores were determined using 2-way ANOVA, with Bonferroni correction for repeated measures of multiple comparisons. Statistical analysis of RNA-seq data is described above. Unless otherwise noted in the figure legends, all other continuous variables were compared using 2-tailed Student's $t$ test with Welch's correction. A $P$ value of $\leq 0.05$ was considered statistically significant.

Study approval. All experiments were performed in accordance with protocols approved by the University of North Carolina Institutional Animal Care and Use Committee (application number 14-001).

\section{Author contributions}

JSS conceived of the project and directed the research; DWB designed all murine experiments and evaluated the data; HES and BRB designed the experiments for the expansion of hILC2s; BGV and DAS performed RNA-seq analysis; DWB, TAD, HB, and SR conducted all murine experiments; JEW and JYPT assisted with permeability assays; JTW scored the histopathology samples; WDS provided the BC-CML tumor cell line; KPM and PMA assisted with flow cytometry experiments; DMWZ and ANJM provided the $\mathrm{Areg}^{-/-}$and $\mathrm{Ill3}^{-/-}$mice, respectively; JMC assist with GFP imaging and design of GVL experiments; and DWB and JSS wrote the manuscript.

\section{Acknowledgments}

This work was supported by NIH grants R01 CA 66794 and R01 HL 115761 (to JSS) and R01 CA 72669 and R01 HL56067 (to BRB) and R37 AI029564 (to JPT). ANJM is supported by the Medical Research Council (U105178805) and Wellcome Trust (100963/Z/13/Z). DWB was supported by NIH grant F32 HL126365. JW was supported by American Cancer Society Postdoctoral Fellowship.

Address correspondence to: Jonathan S. Serody, Marsico Hall, Room 5012, 125 Mason Farm Road, University of North Carolina School of Medicine, Chapel Hill, North Carolina 275997295, USA. Phone: 919.445.4175; E-mail: Jonathan_Serody@ med.unc.edu.
1. Kersey JH. The role of allogeneic-cell transplantation in leukemia. N Engl J Med. 2010;363(22):2158-2159.

2. Horwitz ME. Reduced intensity versus myeloablative allogeneic stem cell transplantation for the treatment of acute myeloid leukemia, myelodysplastic syndrome and acute lymphoid leukemia. Curr Opin Oncol. 2011;23(2):197-202.

3. Faber EA, Vose JM. The role of hematopoietic stem cell transplant in follicular lymphoma. J Natl Compr Canc Netw. 2010;8(3):337-344.

4. Dignan FL, et al. Diagnosis and management of acute graft-versus-host disease. Br J Haematol. 2012;158(1):30-45.

5. Schroeder MA, DiPersio JF. Mouse models of graft-versus-host disease: advances and limitations. Dis Model Mech. 2011;4(3):318-333.

6. Bolaños-Meade J, et al. Phase 3 clinical trial of steroids/mycophenolate mofetil vs steroids/ placebo as therapy for acute GVHD: BMT CTN 0802. Blood. 2014;124(22):3221-3227.

7. Xhaard A, et al. Steroid-refractory acute GVHD: lack of long-term improved survival using new generation anticytokine treatment. Biol Blood Marrow Transplant. 2012;18(3):406-413.

8. Gatti RA, Kersey JH, Yunis EJ, Good RA. Graftversus-host disease. Prog Clin Pathol. 1973;5:1-18.

9. Hülsdünker J, Zeiser R. Insights into the pathogenesis of GvHD: what mice can teach us about man. Tissue Antigens. 2015;85(1):2-9.

10. Magenau J, Runaas L, Reddy P. Advances in understanding the pathogenesis of graft-versushost disease. Br J Haematol. 2016;173(2):190-205.

11. Coghill JM, Sarantopoulos S, Moran TP, Mur- phy WJ, Blazar BR, Serody JS. Effector CD4+ T cells, the cytokines they generate, and GVHD: something old and something new. Blood. 2011;117(12):3268-3276.

12. Highfill SL, et al. Bone marrow myeloid-derived suppressor cells (MDSCs) inhibit graft-versushost disease (GVHD) via an arginase-1-dependent mechanism that is up-regulated by interleukin-13. Blood. 2010;116(25):5738-5747.

13. Klose CS, Artis D. Innate lymphoid cells as regulators of immunity, inflammation and tissue homeostasis. Nat Immunol. 2016;17(7):765-774.

14. Rivera A, Siracusa MC, Yap GS, Gause WC. Innate cell communication kick-starts pathogen-specific immunity. Nat Immunol. 2016;17(4):356-363.

15. Walker JA, Barlow JL, McKenzie AN. Innate lymphoid cells--how did we miss them? Nat Rev Immunol. 2013;13(2):75-87.

16. Anthony RM, Rutitzky LI, Urban JF, Stadecker MJ, Gause WC. Protective immune mechanisms in helminth infection. Nat Rev Immunol. 2007;7(12):975-987.

17. Kim HY, DeKruyff RH, Umetsu DT. The many paths to asthma: phenotype shaped by innate and adaptive immunity. Nat Immunol. 2010;11(7):577-584.

18. Palm NW, Rosenstein RK, Medzhitov R. Allergic host defences. Nature. 2012;484(7395):465-472.

19. Pulendran B, Artis D. New paradigms in type 2 immunity. Science. 2012;337(6093):431-435.

20. Saenz SA, et al. IL-25 simultaneously elicits distinct populations of innate lymphoid cells and multipotent progenitor type 2 (MPPtype2) cells. JExp Med. 2013;210(9):1823-1837.
21. Hanash AM, et al. Interleukin-22 protects intestinal stem cells from immune-mediated tissue damage and regulates sensitivity to graft versus host disease. Immunity. 2012;37(2):339-350.

22. Munneke JM, et al. Activated innate lymphoid cells are associated with a reduced susceptibility to graft-versus-host disease. Blood. 2014;124(5):812-821.

23. Monticelli LA, et al. Innate lymphoid cells promote lung-tissue homeostasis after infection with influenza virus. Nat Immunol. 2011;12(11):1045-1054.

24. Neill DR, et al. Nuocytes represent a new innate effector leukocyte that mediates type- 2 immunity. Nature. 2010;464(7293):1367-1370.

25. Krenger W, Snyder KM, Byon JC, Falzarano G, Ferrara JL. Polarized type 2 alloreactive CD4+ and CD8+ donor T cells fail to induce experimental acute graft-versus-host disease. J Immunol. 1995;155(2):585-593.

26. Zhang Y, Zhang Y, Gu W, Sun B. TH1/TH2 cell differentiation and molecular signals. Adv Exp Med Biol. 2014;841:15-44.

27. Messmann JJ, Reisser T, Leithäuser F, Lutz MB, Debatin KM, Strauss G. In vitro-generated MDSCs prevent murine GVHD by inducing type $2 \mathrm{~T}$ cells without disabling antitumor cytotoxicity. Blood. 2015;126(9):1138-1148.

28. Matte-Martone C, et al. Graft-versus-leukemia (GVL) against mouse blast-crisis chronic myelogenous leukemia (BC-CML) and chronic-phase chronic myelogenous leukemia (CP-CML): shared mechanisms of $\mathrm{T}$ cell killing, but programmed death ligands render CP-CML 
and not BC-CML GVL resistant. J Immunol. 2011;187(4):1653-1663.

29. Coghill JM, et al. CC chemokine receptor 8 potentiates donor Treg survival and is critical for the prevention of murine graft-versus-host disease. Blood. 2013;122(5):825-836.

30. Trenado A, et al. Recipient-type specific $\mathrm{CD} 4+\mathrm{CD} 25+$ regulatory $\mathrm{T}$ cells favor immune reconstitution and control graft-versus-host disease while maintaining graft-versus-leukemia. JClin Invest. 2003;112(11):1688-1696.

31. Esplin BL, Welner RS, Zhang Q, Borghesi LA, Kincade PW. A differentiation pathway for B1 cells in adult bone marrow. Proc Natl Acad Sci US A. 2009;106(14):5773-5778.

32. Mjösberg JM, et al. Human IL-25- and IL-33responsive type 2 innate lymphoid cells are defined by expression of CRTH2 and CD161. Nat Immunol. 2011;12(11):1055-1062.

33. Vély F, et al. Evidence of innate lymphoid cell redundancy in humans. Nat Immunol. 2016;17(11):1291-1299.

34. Björklund ÅK, et al. The heterogeneity of human CD127(+) innate lymphoid cells revealed by single-cell RNA sequencing. Nat Immunol. 2016;17(4):451-460.

35. Lim AI, et al. IL-12 drives functional plasticity of human group 2 innate lymphoid cells. J Exp Med. 2016;213(4):569-583.

36. Ohne Y, et al. IL-1 is a critical regulator of group 2 innate lymphoid cell function and plasticity. Nat Immunol. 2016;17(6):646-655.

37. Bouchery T, et al. ILC2s and T cells cooperate to ensure maintenance of M2 macrophages for lung immunity against hookworms. Nat Commun. 2015;6:6970.

38. Hix LM, Karavitis J, Khan MW, Shi YH, Khaz- aie K, Zhang M. Tumor STAT1 transcription factor activity enhances breast tumor growth and immune suppression mediated by myeloid-derived suppressor cells. J Biol Chem. 2013;288(17):11676-11688.

39. Gabrilovich DI, Ostrand-Rosenberg S, Bronte V. Coordinated regulation of myeloid cells by tumours. Nat Rev Immunol. 2012;12(4):253-268.

40. Condamine T, Gabrilovich DI. Molecular mechanisms regulating myeloid-derived suppressor cel differentiation and function. Trends Immunol. 2011;32(1):19-25.

41. Vander Lugt MT, et al. ST2 as a marker for risk of therapy-resistant graft-versus-host disease and death. N Engl J Med. 2013;369(6):529-539.

42. Ponce DM, et al. High day 28 ST2 levels predict for acute graft-versus-host disease and transplant-related mortality after cord blood transplantation. Blood. 2015;125(1):199-205.

43. Reichenbach DK, et al. The IL-33/ST2 axis augments effector T-cell responses during acute GVHD. Blood. 2015;125(20):3183-3192.

44. Zhang J, et al. ST2 blockade reduces sST2producing $\mathrm{T}$ cells while maintaining protective mST2-expressing T cells during graft-versus-host disease. Sci Transl Med.2015;7(308):308ra160.

45. Zaiss DM, Gause WC, Osborne LC, Artis D. Emerging functions of amphiregulin in orchestrating immunity, inflammation, and tissue repair. Immunity. 2015;42(2):216-226.

46. Arpaia N, et al. A distinct function of regulatory $\mathrm{T}$ cells in tissue protection. Cell. 2015;162(5):1078-1089.

47. Panoskaltsis-Mortari A, et al. In vivo imaging of graft-versus-host-disease in mice. Blood. 2004;103(9):3590-3598.

48. Zaiss DM, Yang L, Shah PR, Kobie JJ, Urban JF,
Mosmann TR. Amphiregulin, a TH2 cytokine enhancing resistance to nematodes. Science. 2006;314(5806):1746.

49. Cancer Genome Atlas Research Network, et al. The Cancer Genome Atlas Pan-Cancer analysis project. Nat Genet. 2013;45(10):1113-1120.

50. Wang K, et al. MapSplice: accurate mapping of RNA-seq reads for splice junction discovery. Nucleic Acids Res. 2010;38(18):e178.

51. Karolchik D, et al. The UCSC Genome Browser database: 2014 update. Nucleic Acids Res. 2014;42(Database issue):D764-D770.

52. Li B, Dewey CN. RSEM: accurate transcript quantification from RNA-Seq data with or without a reference genome. BMC Bioinformatics. 2011;12:323.

53. Bullard JH, Purdom E, Hansen KD, Dudoit S. Evaluation of statistical methods for normalization and differential expression in mRNA-Seq experiments. BMC Bioinformatics. 2010;11:94.

54. Robinette ML, et al. Transcriptional programs define molecular characteristics of innate lymphoid cell classes and subsets. Nat Immunol. 2015;16(3):306-317.

55. Carvalho BS, Irizarry RA. A framework for oligonucleotide microarray preprocessing. Bioinformatics. 2010;26(19):2363-2367.

56. Coghill JM, et al. Separation of graft-versus-host disease from graft-versus-leukemia responses by targeting CC-chemokine receptor 7 on donor $\mathrm{T}$ cells. Blood. 2010;115(23):4914-4922.

57. Fulton LM, et al. Attenuation of acute graft-versushost disease in the absence of the transcription factor ROR $\gamma$ t. JImmunol. 2012;189(4):1765-1772.

58. van Den Brink MR, et al. Fas-deficient lpr mice are more susceptible to graft-versus-host disease. JImmunol.2000;164(1):469-480. 